\title{
Synthetic line and continuum observations of simulated turbulent clouds: the apparent widths of filaments
}

\author{
F. D. Priestley ${ }^{\star}$ and A. P. Whitworth \\ School of Physics and Astronomy, Cardiff University, Queen's Buildings, The Parade, Cardiff CF24 3AA, UK
}

Accepted 2020 October 5. Received 2020 September 17; in original form 2020 August 6

\begin{abstract}
Filamentary structures are ubiquitous in observations of real molecular clouds and also in simulations of turbulent, self-gravitating gas. However, making comparisons between observations and simulations is complicated by the difficulty of estimating volume densities observationally. Here, we have post-processed hydrodynamical simulations of a turbulent isothermal molecular cloud, using a full time-dependent chemical network. We have then run radiative transfer models to obtain synthetic line and continuum intensities that can be compared directly with those observed. We find that filaments have a characteristic width of $\sim 0.1 \mathrm{pc}$, both on maps of their true surface density and on maps of their $850 \mu \mathrm{m}$ dust continuum emission in agreement with previous work. On maps of line emission from CO isotopologues, the apparent widths of filaments are typically several times larger because the line intensities are poorly correlated with the surface density. On maps of line emission from dense gas tracers such as $\mathrm{N}_{2} \mathrm{H}^{+}$and $\mathrm{HCN}$, the apparent widths of filaments are $\lesssim 0.1 \mathrm{pc}$. Thus, current observations of molecular-line emission are compatible with the universal $0.1 \mathrm{pc}$ filament width inferred from Herschel observations, provided proper account is taken of abundance, optical depth, and excitation considerations. We find evidence for $\sim 0.4 \mathrm{~km} \mathrm{~s}^{-1}$ radial velocity differences across filaments. These radial velocity differences might be a useful indicator of the mechanism by which a filament has formed or is forming, for example the turbulent cloud scenario modelled here, as against other mechanisms such as cloud-cloud collisions.
\end{abstract}

Key words: astrochemistry-stars: formation-ISM: clouds - ISM: molecules-ISM: structure.

\section{INTRODUCTION}

Since the observation that far-infrared Herschel images of molecular clouds appear almost ubiquitously filamentary in nature (André et al. 2010), from sub-parsec scales up to $\sim 100 \mathrm{pc}$ giant molecular filaments (Ragan et al. 2014), most studies have accepted that this filamentary structure plays an important role in star formation, for example by controlling the accretion rate on to prestellar cores (Kirk et al. 2013; Peretto et al. 2013) or altering the fragmentation properties of the gas (Sánchez-Monge et al. 2014; Beuther et al. 2015; Ragan et al. 2015). Filaments are commonly produced in simulations of turbulent clouds (Kirk et al. 2015; Federrath 2016) and cloud-cloud collisions (Balfour et al. 2015; Matsumoto, Dobashi \& Shimoikura 2015) or by Galactic shear forces (Duarte-Cabral \& Dobbs 2017; Smith et al. 2020). How much, if anything, each of these mechanisms contributes to the formation of the the observed filaments is an open question, with important implications for our understanding of star formation, both within clouds and on a global scale.

Observationally, Arzoumanian et al. (2011) and Arzoumanian et al. (2019) find that filaments identified in maps of dust continuum intensity appear to have a characteristic width of $\sim 0.1 \mathrm{pc}$, implying that any theoretical or numerical model ought to reproduce this in order to be considered valid. Kirk et al. (2015) and Federrath (2016) both find that simulations of turbulent, self-gravitating clouds produce filaments of this approximate width for typical interstellar

^E-mail: priestleyf@ cardiff.ac.uk medium (ISM) conditions, irrespective of whether magnetic fields are included, but with a somewhat larger range of widths than found by Arzoumanian et al. (2011) and Arzoumanian et al. (2019). However, for simulated filaments, the surface density is known exactly, whereas the observationally inferred surface densities depend on several assumptions about dust properties such as dust temperature, dust optical properties, and gas-to-dust ratio, all of which are poorly constrained and may vary within the filament (e.g. Ossenkopf \& Henning 1994; Howard et al. 2019). If molecular-line radiation is used to define filaments (rather than dust continuum emission), the inferred filament widths depend on the molecule oberved, from $\sim 0.01 \mathrm{pc}$ for $\mathrm{N}_{2} \mathrm{H}^{+}$(Hacar et al. 2018) to $0.4 \mathrm{pc}$ for ${ }^{13} \mathrm{CO}$ (Panopoulou et al. 2014), although Suri et al. (2019) find a value in agreement with Arzoumanian et al. (2011) using $\mathrm{C}^{18}$ O. Smith, Glover \& Klessen (2014) and Panopoulou et al. (2017) have also raised concerns about the effect of the fitting method on estimates of filament width. It is therefore unclear whether the correspondence between observed and simulated filament widths is really showing that the simulations are correct.

The above issues can be mitigated by converting the output of hydrodynamical simulations into synthetic observations of commonly detected molecules. These can then be compared directly with observed filaments. Previous work has largely focused on emission from [C II] and CO isotopologues (e.g. Franeck et al. 2018; Peñaloza et al. 2018), the abundances of which are already calculated in many simulations in order to determine cooling rates, on scales from giant molecular clouds (Duarte-Cabral \& Dobbs 2016) to individual star-forming filaments (Clarke et al. 2018). However, the 
chemical networks used (e.g. Glover \& Clark 2012) often neglect freeze-out of $\mathrm{CO}$ on to dust grains, which can significantly reduce its abundance in the densest regions; within these regions, even the rarest isotopologues may also be optically thick. Smith et al. (2012) and Smith et al. (2013) investigate emission from other molecular species but assume an abundance for each species, rather than calculating it self-consistently. In this paper, we track the full time-dependent chemical evolution of a turbulent filamentary cloud, allowing us to generate synthetic line emission maps for any molecule in our chemical network. By computing the emergent intensities of many different molecular lines, tracing a range of gas densities, and then comparing them with the observed intensites, we are able to significantly improve constraints on the structure of star-forming filaments.

\section{METHOD}

We model the hydrodynamical evolution of a turbulent molecular cloud using the Smoothed Particle Hydrodynamics (SPH) code PHANTOM (Price et al. 2018). We consider a uniform density spherical cloud of mass $M=200 \mathrm{M}_{\odot}$ and radius $R=1 \mathrm{pc}$, giving an initial density of $3.23 \times 10^{-21} \mathrm{~g} \mathrm{~cm}^{-3}\left(\equiv 680 \mathrm{H}_{2} \mathrm{~cm}^{-3}\right)$. The gas in the cloud is isothermal at $T=10 \mathrm{~K}$ and the hydrogen is molecular; hence, the isothermal sound speed is $c_{\mathrm{s}}=0.2 \mathrm{~km} \mathrm{~s}^{-1}$. The cloud is at the centre of a cubic box with side length $4 R$. Outside the cloud, the density is $3.23 \times 10^{-23} \mathrm{~g} \mathrm{~cm}^{-3}\left(\equiv 6.8 \mathrm{H}_{2} \mathrm{~cm}^{-3}\right)$, the gas is isothermal at $T=1000 \mathrm{~K}$, and the hydrogen is molecular; hence, the isothermal sound speed is $c_{\mathrm{s}}=2 \mathrm{~km} \mathrm{~s}^{-1}$. There is therefore pressure balance across the boundary of the cloud. A turbulent velocity field is generated with a power spectrum $P(k) \propto k^{-4}$, wavenumbers between $k_{\min }=1$ and $k_{\max }=100$ (where $k_{\min }=1$ corresponds to wavelength $\lambda=R$ ), and a thermal mix of compressive and solenoidal modes. The SPH-particle velocities are scaled so that the initial root-mean-squared Mach number is 5. These values give similar turbulent velocity fields to those simulated by Kirk et al. (2015) and Federrath (2016), although some details, such as the implementation of turbulence, differ. We use $\sim 140000$ particles in the cloud, and a further $\sim 20000$ outside the cloud, with a particle mass resolution of $1.4 \times 10^{-3} \mathrm{M}_{\odot}$. The mean number of neighbours within an SPH smoothing kernel is 57 . Increasing the mass resolution by a factor of 5 , by increasing the number of SPH particles to $\sim 800000$, does not noticeably change the results.

For isothermal gas where hydrogen is already in molecular form, the chemical evolution is almost completely decoupled from the hydrodynamics. We can therefore post-process our SPH results with a time-dependent chemical code, as in Priestley, Wurster \& Viti (2019). We use a subset of $10000 \mathrm{SPH}$ particles, ${ }^{1}$ chosen randomly from those with initial positions inside the cloud, and input their density evolution as a function of time into UCL_CHEM (Holdship et al. 2017), which uses the UMIST12 reaction network (McElroy et al. 2013) with additional molecular freeze-out and grain surface reactions as described in Holdship et al. (2017). For all SPH particles, we assume a constant gas-kinetic temperature ( $T=10 \mathrm{~K}$ for those in the cloud, and $T=1000 \mathrm{~K}$ for those outside the cloud), a dust temperature $T_{\text {dust }}=10 \mathrm{~K}$, a cosmic ray ionization rate per $\mathrm{H}_{2}$ molecule of $1.3 \times$ $10^{-17} \mathrm{~s}^{-1}$, and elemental abundances from Lee et al. (1998).

\footnotetext{
${ }^{1}$ This means that even SPH particles whose chemical evolution is not followed are likely to have several (on average 6) SPH particles within their smoothing kernel whose chemical evolution is followed.
}

In some previous work on prestellar cores, the visual extinction $A_{\mathrm{V}}$ at each point in the cloud has either been calculated exactly exploiting imposed symmetries (e.g. Aikawa et al. 2005; Tassis et al. 2012; Priestley, Viti \& Williams 2018), or has been assumed to be large enough, due to external shielding, to make the radiation field negligible (Priestley et al. 2019). The former situation does not apply to turbulent clouds, while on scales of $\gtrsim 1 \mathrm{pc}$, the second is not justifiable either. Several algorithms have been developed to estimate the shielding column density (and hence $A_{\mathrm{V}}$ ) in hydrodynamical simulations (e.g. Clark, Glover \& Klessen 2012). However, these still entail significant computational expense to obtain useful resolution. Here, we approximate $A_{\mathrm{V}}$ using a modified version of the method of Dobbs et al. (2008), who assumed that the effective column density is just the local density multiplied by a length scale. While those authors used a constant length scale, we instead use the local Jeans length, defined as $c_{\mathrm{s}}(G \rho)^{-1 / 2}$, which we find returns column densities comparable to the maximum midplane value for the densest gas. We convert the column density to $A_{\mathrm{V}}$ with a typical ISM factor of $6.0 \times 10^{-22} \mathrm{mag}\left(\mathrm{H} \mathrm{cm}^{-2}\right)^{-1}$ (Bohlin, Savage \& Drake 1978). We investigate ambient far-ultraviolet (FUV) radiation field strengths of $G=0,1$, and 5 measured in Habing units (Habing 1968). $G=1$ is the fiducial case, on which we focus the discussion.

Our chemical modelling gives us the detailed three-dimensional chemical composition of the cloud at each point in time during the simulation. We use LIME (Brinch \& Hogerheijde 2010) to convert this into synthetic dust continuum and molecular-line observations, using dust optical properties from Ossenkopf \& Henning (1994) and molecular data from the LAMDA data base (Schöier et al. 2005). We use 10000 randomly distributed sample points, assigning each point the density, molecular abundance, and velocity of the nearest SPH particle. While this undersamples denser regions, compared to the distribution of SPH particles, we find that increasing the resolution does not substantially change our results. We generate images in dust continuum emission, and in various molecular lines, as observed along the coordinate axes (i.e. projected on the $x=0, y=0$, and $z=$ 0 planes), and compare these with the true surface density, e.g. for images projected on the $y=0$ plane

$\Sigma(x, z)=\int_{\mathrm{y}=-2 R}^{\mathrm{y}=+2 R} \rho(x, y, z) d y$.

We use a pixel size of $0.005 \mathrm{pc}$, comparable to the typical SPH smoothing length. We identify filaments using the DisPerSE algorithm (Sousbie 2011). The persistence threshold required by DisPerSE is computed from the intensities outside a projected radius of $R$, which do not contain any cloud particles and therefore represent a 'background' noise level. Our chemical network does not distinguish between isotopes of the same element and ignores chemical fractionation; when calculating the emission from isotopologues, we assume ${ }^{12} \mathrm{C} /{ }^{13} \mathrm{C}=100$ and ${ }^{16} \mathrm{O} /{ }^{18} \mathrm{O}=500$.

\section{RESULTS}

Fig. 1 shows images on the $y=0$ plane of the surface density, the monochromatic intensity of dust continuum emission at $850 \mu \mathrm{m}$, and the integrated intensities of the $J=1-0$ lines from ${ }^{13} \mathrm{CO}$ and $\mathrm{HCN}$, for the $G=1$ case after $0.868 \mathrm{Myr}$, at which point, the range of surface densities is comparable to the models presented in Federrath (2016). One can identify by eye filamentary structures in the surface density image, and the dust continuum emission also traces the surface density accurately. There is much lower contrast between lower and higher surface density gas in the ${ }^{13} \mathrm{CO}$ line emission, and, consequently, this emission map appears more lumpy 

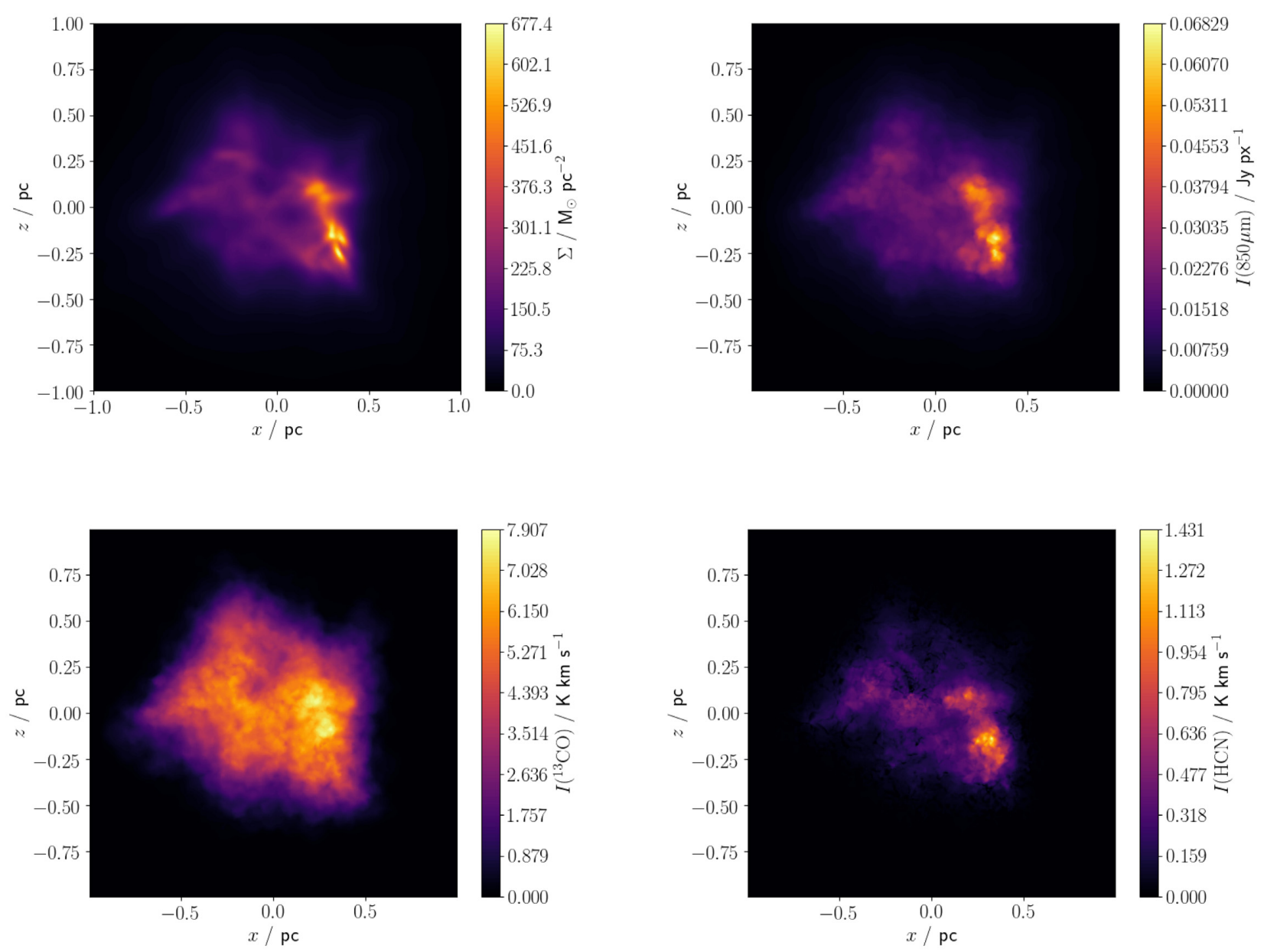

Figure 1. Images projected on the $y=0$ plane for the $G=1$ case at $0.868 \mathrm{Myr}$. Top left: surface density. Top right: monochromatic intensity of dust continuum emission at $850 \mu \mathrm{m}$. Bottom left: integrated intensity of the ${ }^{13} \mathrm{CO}(J=1-0)$ line. Bottom right: integrated intensity of the $\mathrm{HCN}(J=1-0)$ line.
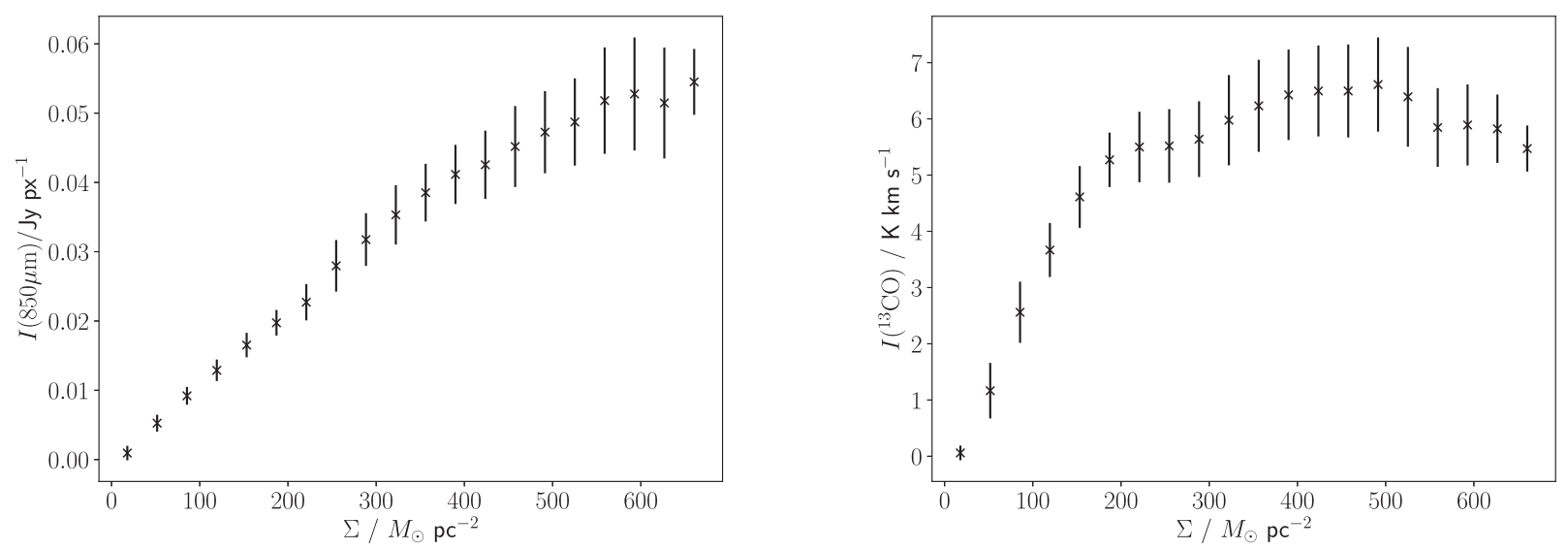

Figure 2. The ratio of intensity to surface density on the $y=0$ plane for the $G=1$ case at $0.868 \mathrm{Myr}$. Left: monochromatic intensity of dust continuum emission at $850 \mu \mathrm{m}$. Right: integrated line intensity of ${ }^{13} \mathrm{CO} \mathrm{J}=1-0$ emission. The points give mean values, and the error bars give standard deviations.

than filamentary. The HCN emission is limited to the highest density regions.

This can be seen more clearly in the ratios of intensity to surface density, shown in Fig. 2. The $850 \mu \mathrm{m}$ intensity increases approximately in proportion to the surface density up to $\Sigma \sim 500 \mathrm{M}_{\odot} \mathrm{pc}^{-2}$, but above this value, the $850 \mu \mathrm{m}$ intensity is somewhat underestimated. This is because the surface density maps are evaluated using SPLASH (Price 2007), which uses all the SPH particles and an accurate interpolation procedure; in contrast, the $850 \mu \mathrm{m}$ dust emission maps produced by LIME have lower resolution, because (a) they use only the chemical tracer particles, and (b) LIME adopts a much cruder interpolation procedure to obtain the dust density along the line of sight. The ${ }^{13} \mathrm{CO} J=1-0$ integrated intensity 'saturates' at $\Sigma \sim 250 \mathrm{M}_{\odot} \mathrm{pc}^{-2}$, because it becomes optically thick, and for 

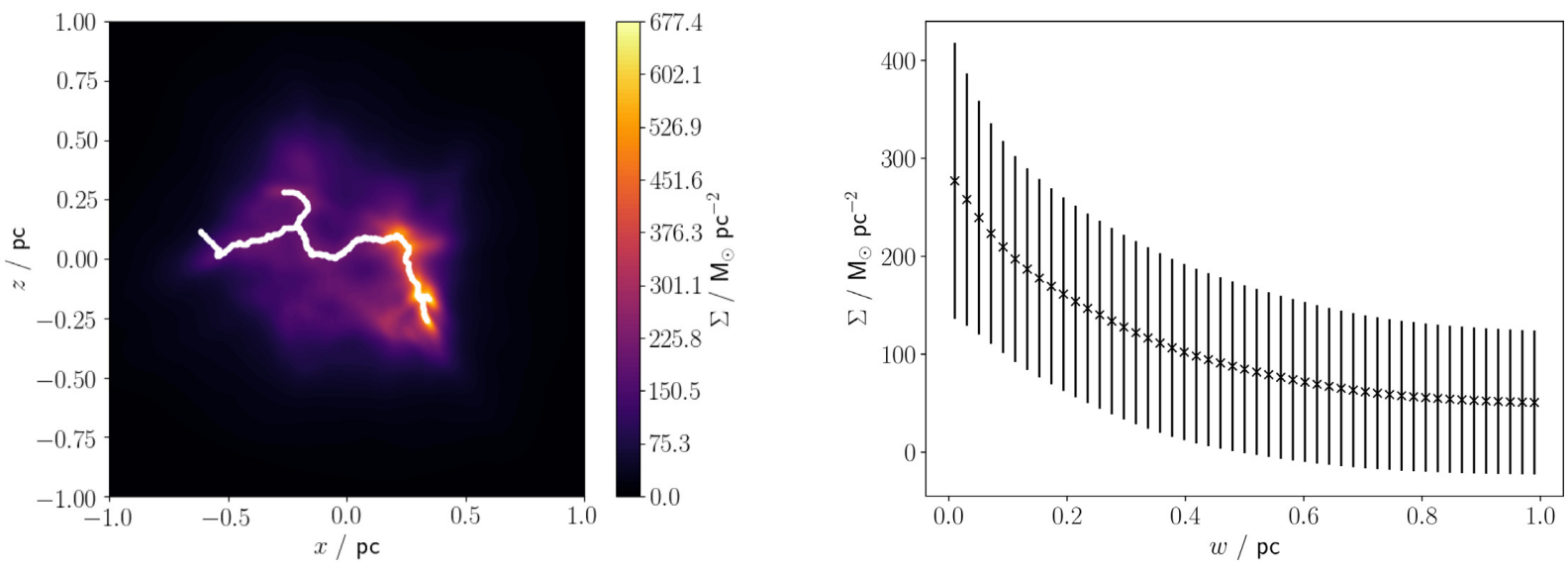

Figure 3. The $G=1$ case at $0.868 \mathrm{Myr}$, projected on the $y=0$ plane. Left: surface density image with white pixels tracing the filament spine as identified by DisPerSE. Right: average surface density profile, $\Sigma(w)$, perpendicular to the filament; the points give mean values, and the error bars give standard deviations.

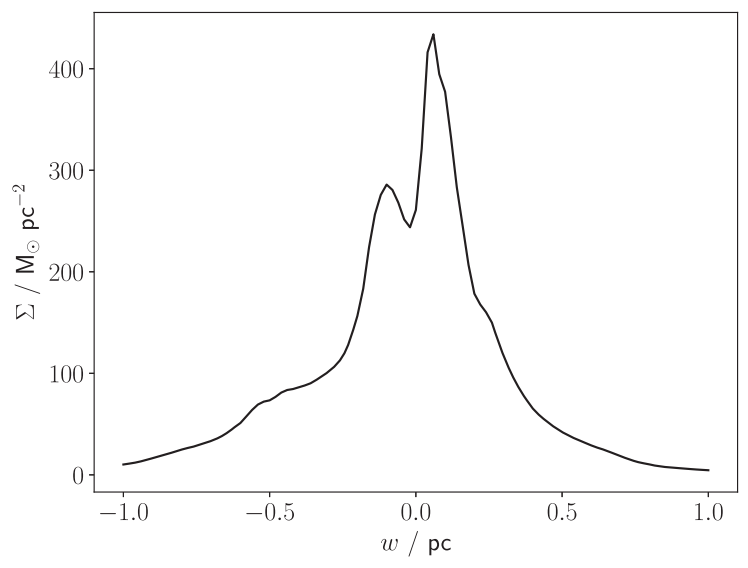

Figure 4. Average surface density profile perpendicular to the filamentary structure traced by DisPerSE from the surface density map on the $y=0$ plane for the $G=1$ case at $0.868 \mathrm{Myr}$.

$\Sigma \gtrsim 500 \mathrm{M}_{\odot} \mathrm{pc}^{-2}$, it declines due to freeze-out of ${ }^{13} \mathrm{CO}$. Despite this lack of sensitivity to high densities, the filamentary spines traced by DisPerSE for both the dust $850 \mu \mathrm{m}$ and the ${ }^{13} \mathrm{CO} J=1-0$ intensity maps are very similar to the actual surface density map. For consistency, we use the filament spine traced using the surface density map (as shown in the left-hand panel of Fig. 3) to determine filament properties in all observational tracers.

The right-hand panel of Fig. 3 shows the mean surface density profile perpendicular to the filament spine, $\bar{\Sigma}_{\perp}(w)$, where $w$ is the impact parameter relative to the spine, and the averaging is over all the pixels defining the spine. To calculate this profile, we identify for each general pixel, $p$, the closest spine pixel, $s$, and compute the distance between them, $w_{\text {sp }}$. The surface density, $\Sigma_{p}$, at general pixel $p$ then contributes to the surface density profile perpendicular to the filament at spine pixel $s$, i.e. $\Sigma_{\perp s}\left(w_{\text {sp }}\right)=\Sigma_{p}$. The mean surface density profile $\bar{\Sigma}_{\perp}(w)$ is obtained by averaging $\Sigma_{\perp s}(w)$ over all spine pixels, s. Whereas Federrath (2016) find sharply peaked profiles with a well-defined typical full width at half-maximum (FWHM) of $\sim 0.1 \mathrm{pc}$, our profiles are much broader, with mean FWHM $\sim 0.6 \mathrm{pc}$ and significant scatter around the mean at all impact parameters. This is a consequence of the averaging process. For an individual filament spine pixel, as identified by DisPerSE, the surface density profile may have multiple, off-centre peaks, as illustrated by Fig. 4. These individual peaks have widths comparable to those seen in Federrath (2016), but the effect of averaging all points together results in a much broader distribution. A very similar phenomenon has been found by Suri et al. (2019) in $\mathrm{C}^{18} \mathrm{O}$ observations of the Orion A molecular cloud, who show that the width of the average filament profile is much broader than the typical width of the individual density peaks.

We note that although many of our simulation parameters are very similar to those of Federrath (2016), there are some significant differences: our simulation includes only an initial turbulent velocity field, which is then allowed to decay, as opposed to continuously driven turbulence, and we consider an isolated cloud, rather than a periodic box.

Filament widths are usually estimated by fitting an assumed functional form to the profile perpendicular to the filament spine (Arzoumanian et al. 2011; Federrath 2016; Suri et al. 2019), most commonly a Plummer-like profile,

$\Sigma(w)=\Sigma_{\mathrm{o}}\left\{1+\left(\frac{w}{w_{\mathrm{O}}}\right)^{2}\right\}^{-p / 2}$,

where $\Sigma_{\mathrm{O}}$ is the value on the spine, $w_{\mathrm{O}}$ is the scale length close to the spine, and $p$ is the envelope exponent (Whitworth \& Ward-Thompson 2001). As the profiles in our simulations are not necessarily well represented by this (or any other) functional form, and in some instances are not even particularly symmetrical, we instead measure the widths by calculating the half width at half maximum (HWHM), separately on each side of the filament spine. Fig. 5 shows the distributions of HWHMs for the $G=1$ case, estimated on the basis of the actual surface density and on the basis of the intensities of $850 \mu \mathrm{m}$ dust continuum emission, ${ }^{13} \mathrm{CO}(1-0)$ line emission, and $\mathrm{N}_{2} \mathrm{H}^{+}$(1-0) line emission. The corresponding median widths, and the 16th and 84th percentiles, are presented in Table 1. In the fiducial case, $G=1$, the distributions of HWHMs for the actual surface density and for the $850 \mu \mathrm{m}$ intensity are quite broad, with median at $\sim 0.17 \mathrm{pc}$.

The median HWHMs from our surface density and dust continuum maps are larger than the full widths obtained both observationally (e.g. Arzoumanian et al. 2011) and from previous simulations (e.g. Federrath 2016), suggesting that our filaments are significantly wider. In fact, these authors refer to the FWHMs of fits to the filament profiles, rather than those of the profiles themselves. The fit FWHM is often smaller by a factor of up to a few than that of the profile itself, and for asymmetric, multiply peaked profiles such as the example 

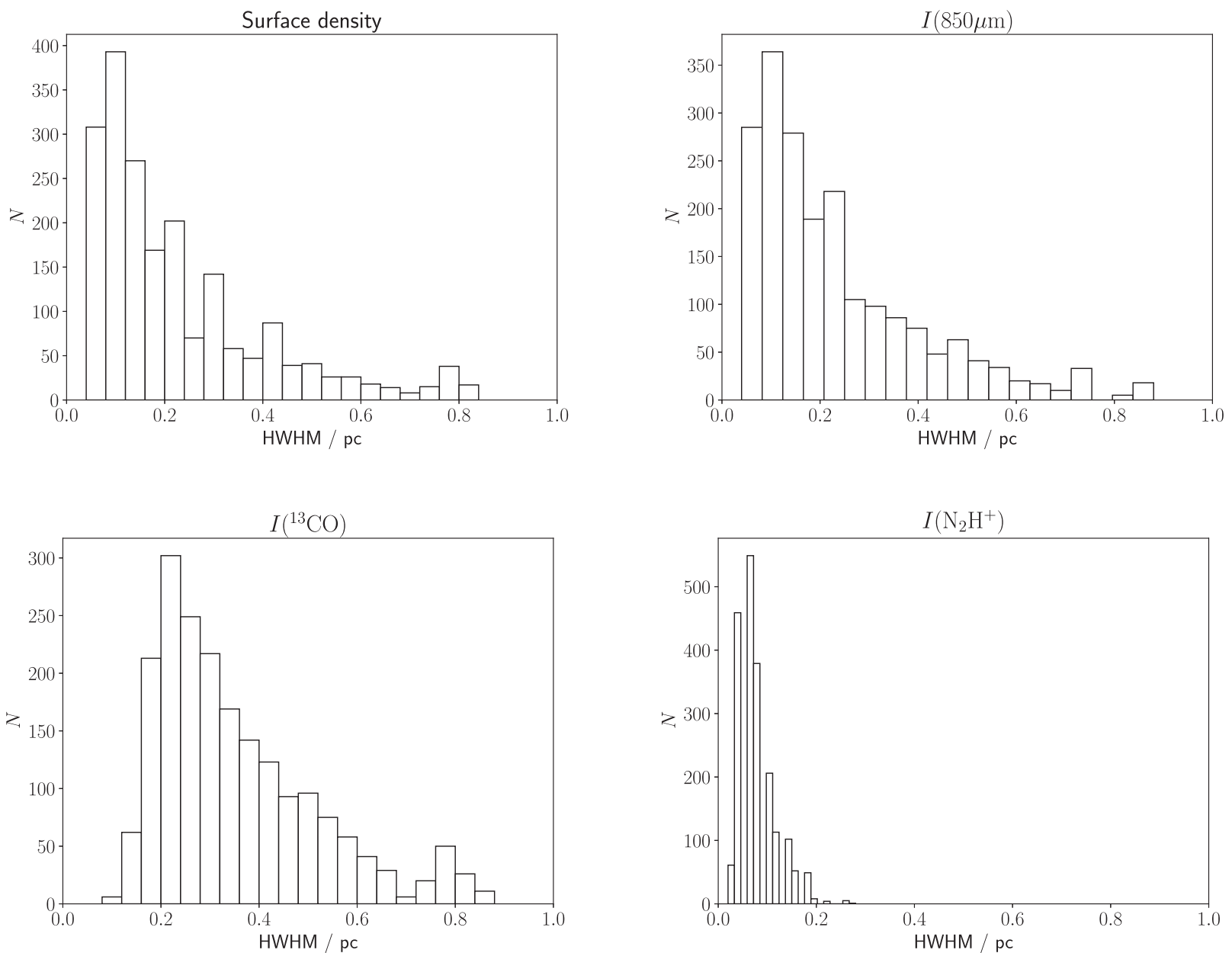

Figure 5. Distributions of filament HWHMs for the $G=1$ case at $0.868 \mathrm{Myr}$, derived from maps of the actual surface density (top left), the $850 \mu \mathrm{m}$ dust continuum intensity (top right), the ${ }^{13} \mathrm{CO}(1-0)$ intensity (bottom left), and the $\mathrm{N}_{2} \mathrm{H}^{+}$(1-0) intensity (bottom right).

Table 1. Filament HWHM median values and 16th and 84th percentiles after $0.868 \mathrm{Myr}$, in pc, for differing radiation field strengths, $G$, based on surface density, dust continuum, and various molecular-line intensities.

\begin{tabular}{cccccccccc}
\hline$G$ & & $\Sigma$ & $I(850 \mu \mathrm{m})$ & ${ }^{13} \mathrm{CO}$ & $\mathrm{C}^{18} \mathrm{O}$ & $\mathrm{NH}_{3}$ & $\mathrm{HCN}$ & $\mathrm{HCO}^{+}$ & $\mathrm{N}_{2} \mathrm{H}^{+}$ \\
\hline 0 & Median & 0.16 & 0.18 & 0.34 & 0.26 & 0.18 & 0.10 & 0.12 & 0.12 \\
& Range & $0.08-0.40$ & $0.10-0.40$ & $0.22-0.56$ & $0.16-0.48$ & $0.12-0.32$ & $0.06-0.16$ & $0.08-0.22$ & $0.06-0.22$ \\
\multirow{2}{*}{1} & Median & 0.16 & 0.18 & 0.30 & 0.24 & 0.12 & 0.12 & 0.10 & 0.06 \\
& Range & $0.08-0.40$ & $0.10-0.40$ & $0.20-0.52$ & $0.14-0.44$ & $0.08-0.20$ & $0.06-0.20$ & $0.06-0.20$ & $0.04-0.12$ \\
5 & Median & 0.16 & 0.18 & 0.26 & 0.20 & 0.10 & 0.10 & 0.08 & 0.06 \\
& Range & $0.08-0.40$ & $0.10-0.40$ & $0.16-0.44$ & $0.12-0.38$ & $0.06-0.18$ & $0.06-0.18$ & $0.06-0.14$ & $0.04-0.10$
\end{tabular}

shown in Fig. 4 this discrepancy can be even greater. If we instead estimate widths by fitting Plummer-like profiles to the region between the closest minima on either side of a peak, as in Suri et al. (2019), we obtain a much narrower distribution centred at smaller values, as found by previous authors. However, even with this procedure, the result depends sensitively on whether the envelope exponent for the Plummer-like fit, $p$ (see equation 2), is fixed or allowed to vary. Fixing $p$ often produces poor fits, while treating $p$ as a free parameter sometimes produces unphysical values for the scale length, $w_{\mathrm{o}}$. Moreover, these issues tend to be more severe for line emission, because the intensity profiles are usually much less smooth. We shall therefore continue to use the HWHM to estimate filament widths. While the exact values of filament widths depend on the procedure used to extract them, it is clear that there is a characteristic width of order $\sim 0.1 \mathrm{pc}$, and that it is well traced by the dust continuum.

HWHMs based on maps of molecular-line emission are sometimes highly dependent on the strength of the ambient FUV radiation field, $G$, because this affects the abundances of the emitting molecules. The stronger the ambient FUV radiation field, the more certain molecules become confined to the dense, well-shielded inner parts of a filament, and consequently the narrower the filament appears to be when estimated using line radiation from such molecules. Conversely, the weaker the ambient FUV radiation field, the more extensively the molecule is distributed, and the wider the estimated HWHM. This effect is particularly notable for lines with a relatively low critical excitation density. 

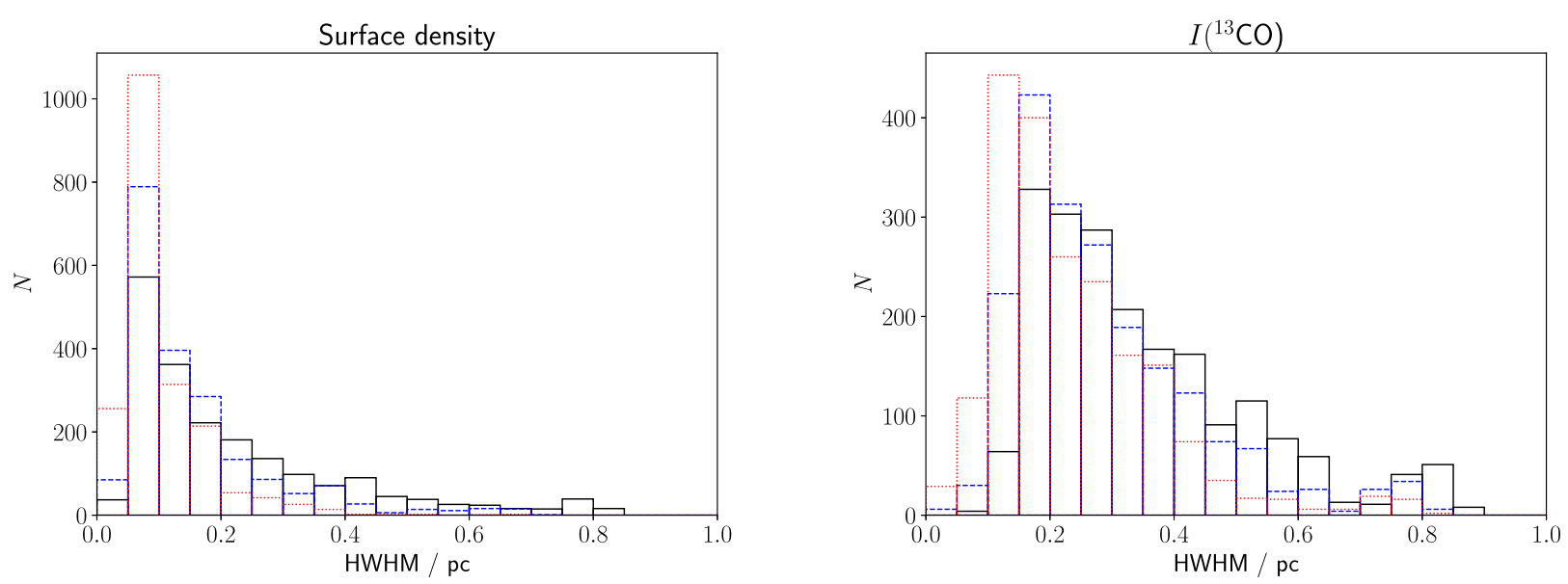

Figure 6. Distributions of filament HWHMs for the $G=1$ case at $0.868 \mathrm{Myr}$, derived from maps of the actual surface density $(l e f t)$ and the ${ }^{13} \mathrm{CO}(1-0)$ intensity (right), with different levels of background subtraction. Left: no background subtraction (black solid line), $50 \mathrm{M}_{\odot} \mathrm{pc}^{-2}$ background (blue dashed line), and $100 \mathrm{M}_{\odot} \mathrm{pc}^{-2}$ background (red dotted line). Right: no background subtraction (black solid line), $1 \mathrm{~K} \mathrm{~km} \mathrm{~s}^{-1}$ background (blue dashed line), and $2 \mathrm{~K} \mathrm{~km} \mathrm{~s}^{-1}$ background (red dotted line).

For example, in the fiducial case, $G=1$, HWHMs based on maps of ${ }^{13} \mathrm{CO}(1-0)$ or $\mathrm{C}^{18} \mathrm{O}(1-0)$ line emission are significantly larger than those based on maps of surface density or dust continuum emission. Median values are $\sim 0.30 \mathrm{pc}$ for ${ }^{13} \mathrm{CO}(1-0)$ and $\sim 0.24 \mathrm{pc}$ for $\mathrm{C}^{18} \mathrm{O}$ (1-0), similar to the values seen by Panopoulou et al. (2014) in the Taurus molecular cloud. However, if the FUV radiation field is increased to $G \gtrsim 5$, HWHMs based on maps of ${ }^{13} \mathrm{CO}(1-0)$ or $\mathrm{C}^{18} \mathrm{O}$ (1-0) line emission become comparable with those based on maps of surface density or dust continuum emission, because ${ }^{13} \mathrm{CO}$ and $\mathrm{C}^{18} \mathrm{O}$ then have very low abundance outside the dense inner regions of the filament. This may explain why Suri et al. (2019) and Orkisz et al. (2019) find that in Orion, where $G \gtrsim 5$, HWHMs based on maps of $\mathrm{C}^{18} \mathrm{O}$ (1-0) agree well with those based on dust emission.

HWHMs based on maps of line emission from molecules like $\mathrm{N}_{2} \mathrm{H}^{+}$, which have higher critical excitation densities and are therefore perceived as dense-gas tracers, are much lower than those from $\mathrm{CO}$ isotopologues, more tightly peaked, and on average even lower than HWHMs based on maps of surface density. This is largely due to the rapid decline in volume emissivity with decreasing volume density, leading to a minimal contribution from foreground and background diffuse gas; there may also be a small decrease in HWHM due to reduced abundance if the FUV radiation field is higher.

Although dense gas tracers give filament widths $\lesssim 0.1 \mathrm{pc}$, our values are still larger than those found by Hacar et al. (2018), $\lesssim 0.02$ pc, using $\mathrm{N}_{2} \mathrm{H}^{+}$observations. However, Hacar et al. (2018) were measuring intertwined and approximately parallel 'fibres' within a larger filamentary structure. Our simulations do not have sufficient resolution to distinguish such fibres, with a typical smoothing length within the filaments of $0.01 \mathrm{pc}$, and the effect of smearing them together would be to produce a single filament with width comparable to the estimates we obtain with $G \gtrsim 1$.

A potential issue with our method of determining filament widths is that we assume that all the emission/surface density is filamentary, without accounting for background contributions from the more diffuse material making up the cloud. In Fig. 6, we show the effect of subtracting increasingly stringent background values from the surface density and ${ }^{13} \mathrm{CO}$ intensity on the filament width distributions, up to $100 \mathrm{M}_{\odot} \mathrm{pc}^{-2}$ and $2 \mathrm{~K} \mathrm{~km} \mathrm{~s}^{-1}$, respectively. This reduces both the median and the range of the width distributions but does not alter our conclusions. For a $2 \mathrm{~K} \mathrm{~km} \mathrm{~s}^{-1}$ background, the ${ }^{13} \mathrm{CO}$ width

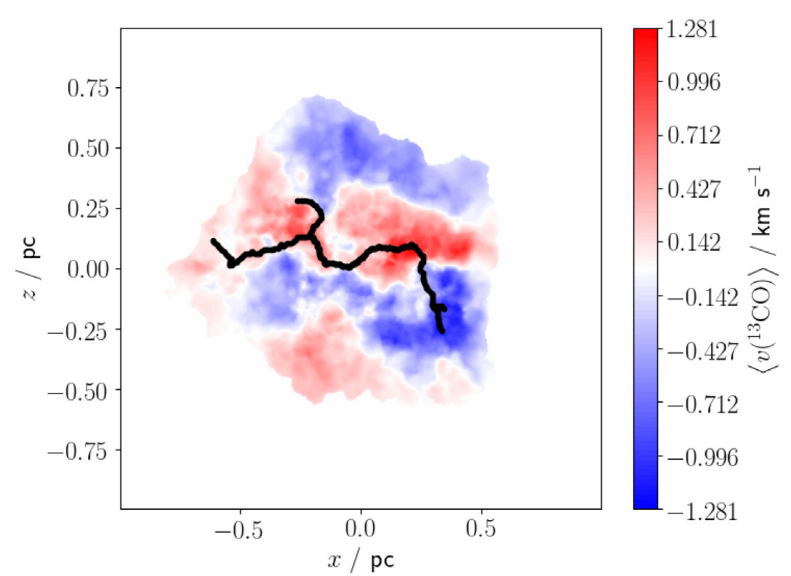

Figure 7. Intensity-weighted ${ }^{13} \mathrm{CO}$ velocity map for the $G=1$ case at $0.868 \mathrm{Myr}$, with the filament spine pixels coloured black.

distribution is still centred at significantly larger values $(0.2 \mathrm{pc})$ than that of the unsubtracted surface density, and for the highest value we consider, the median surface density filament width is reduced to $0.08 \mathrm{pc}$. Similar results are obtained for other tracers: while the choice of background level can reduce the obtained characteristic width, the relative values between tracers are not greatly affected as long as consistent background levels are chosen. Even if a fairly high background level is assumed for some tracers while no subtraction is made for others, it is difficult to disrupt the hierarchy in Table 1 of, for example, $\mathrm{HWHM}_{13} \mathrm{CO}>\mathrm{HWHM}_{\Sigma}>\mathrm{HWHM}_{\mathrm{HCN}}$.

Fig. 7 shows the first-moment map of the ${ }^{13} \mathrm{CO}(1-0)$ intensity for the $G=1$ case at $0.868 \mathrm{Myr}$, projected on to the $y=0$ plane (i.e. the ${ }^{13} \mathrm{CO}$ intensity weighted mean $y$-component of velocity, $v_{y}$ ). The pixels defining the spine of the filament (as identified by DisPerSE) are black and tend to be located between approaching and receding gas, i.e. where there is a marked radial velocity difference across the filament. Projections on to the $z=0$ and $x=0$ planes show a similar behaviour - as do the maps for other molecular lines, although, due to the lower number of pixels with appreciable emission, this is less obvious for molecules like $\mathrm{N}_{2} \mathrm{H}^{+}$. 


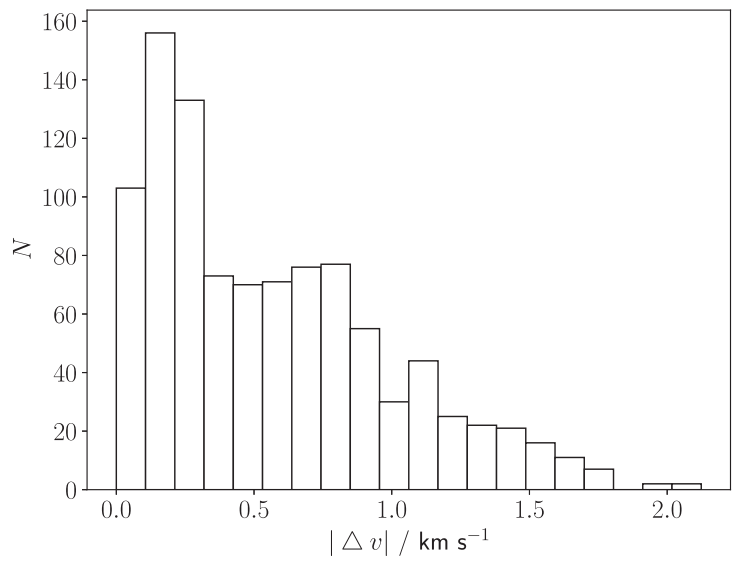

Figure 8. Distribution of ${ }^{13} \mathrm{CO}$ radial velocity differences across the filament for the $G=1$ case at $0.868 \mathrm{Myr}$.

We estimate the radial velocity difference according to,

$\Delta v_{s}=\left|v_{\mathrm{R}}\left(\boldsymbol{r}_{s}+\mathrm{HWHM}_{s}^{+} \hat{\boldsymbol{n}}_{s}\right)-v_{\mathrm{R}}\left(\boldsymbol{r}_{s}-\mathrm{HWHM}_{s}^{-} \hat{\boldsymbol{n}}_{s}\right)\right|$,

for each spine pixel. In equation (3), $v_{\mathrm{R}}$ is the ${ }^{13} \mathrm{CO}$ intensityweighted mean radial velocity, $\boldsymbol{r}_{s}$ is the position of pixel $s$ on the $y=0$ plane, $\hat{\boldsymbol{n}}_{s}$ is the unit normal to the filament spine at pixel $s, \mathrm{HWHM}_{s}^{+}$is the HWHM of the filament at pixel $s$ on the positive side (as defined by the unit normal, $\hat{\boldsymbol{n}}_{s}$ ), and $\mathrm{HWHM}_{s}^{-}$is the HWHM of the filament at pixel $s$ on the negative side. Fig. 8 shows the distribution of radial velocity differences for the $G=1$ case at $0.868 \mathrm{Myr}$. The distribution is peaked at $\sim 0.20 \mathrm{~km} \mathrm{~s}^{-1}\left(\sim c_{\mathrm{s}}\right)$, but with a substantial tail at higher values. The median is $0.48 \mathrm{~km} \mathrm{~s}^{-1}$, and the 16th and 84th percentiles are 0.14 and $1.0 \mathrm{~km} \mathrm{~s}^{-1}$, respectively. Analysing the plane-of-sky velocities of simulated filaments, Smith et al. (2016) reached similar conclusions - filaments tend to be moving supersonically with respect to the surrounding gas, as a result of forming at the convergence points of the turbulent velocity field.

Position-velocity analysis is potentially a means of constraining the mechanisms regulating the formation and evolution of molecular clouds (e.g. Haworth et al. 2015). In the present context, the radial velocity difference across a filament may be a discriminating signature of filament formation by turbulence (as here). The data presented in Ragan et al. (2012) and Watkins et al. (2019) show some indication for velocity gradients perpendicular to the filament long axes, but on

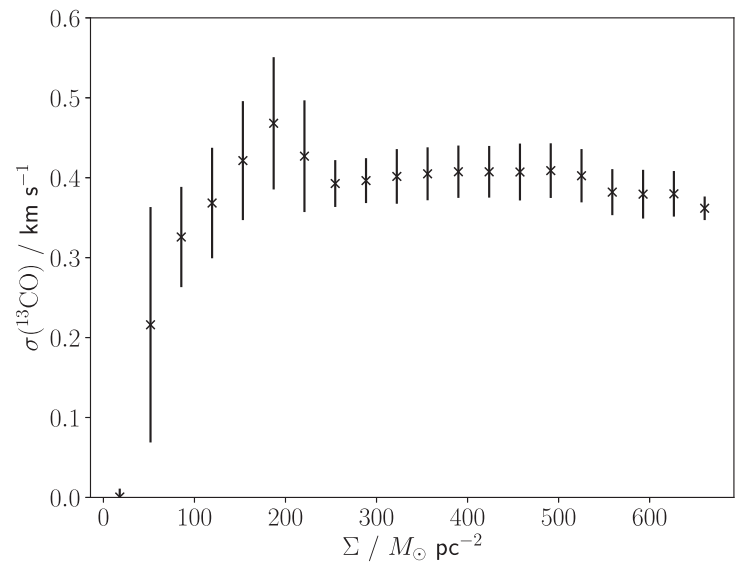

larger scales than those simulated here, and rotation would produce a similar effect. Arzoumanian et al. (2018) find evidence for redand blue-shifted ${ }^{13} \mathrm{CO}$ emission on alternate sides of a $\sim 1 \mathrm{pc}$-long filament identified in $\mathrm{C}^{18} \mathrm{O}$, which they attributed to interaction between the filament and an extended sheet-like structure. This is more comparable to the situation in our simulations, being of a similar size and clearly indicating large-scale motions of less dense material, rather than rotation in the filament itself. Whether this phenomenon is widespread, and whether it can be produced by models other than the fragmentation of a turbulent cloud, is deserving of future study.

Fig. 9 shows how the radial velocity dispersion varies with surface density for the $G=1$ case at $0.868 \mathrm{Myr}$. The left plot uses the ${ }^{13} \mathrm{CO}(1-0)$ line, and the right plot uses the $\mathrm{HCN}$ (1-0) line. At low surface density, $\Sigma<200 \mathrm{M}_{\odot} \mathrm{pc}^{-2}$, in the outer parts of the cloud, the turbulence has decayed and the velocity dispersion is by this stage very low. At higher surface densities, $\Sigma \gtrsim 200 \mathrm{M}_{\odot} \mathrm{pc}^{-2}$, where the bulk of the gas is, the velocity dispersion is essentially independent of surface density, at $0.3-0.4 \mathrm{~km} \mathrm{~s}^{-1}$, i.e. mildly transsonic. There is a slight tendency for dense gas tracers like $\mathrm{HCN}$ to display somewhat lower dispersions than $\mathrm{CO}$ isotopologues and $\mathrm{NH}_{3}$. Assuming, based on Fig. 1, that the structures traced by $\mathrm{HCN}$ and ${ }^{13} \mathrm{CO}$ are approximately 0.5 and $1 \mathrm{pc}$ in size, respectively, the linewidth-size relation (Larson 1981) predicts a velocity dispersion ratio of $\sim 0.75$ for an exponent of 0.4 in good agreement with the actual values $\left(\sim 0.3\right.$ and $0.4 \mathrm{~km} \mathrm{~s}^{-1}$, respectively). These velocity dispersions are in good agreement with the observational results of Arzoumanian et al. (2013) and Hacar et al. (2018).

\section{DISCUSSION}

As with many previous studies, we find that the filaments that form in our simulations have a characteristic width of $\sim 0.1 \mathrm{pc}$. We have also shown that the far-IR dust emission is a reliable tracer of the gas surface density. Since we assume a constant universal dust temperature and constant universal dust properties, this is not surprising, since the dust emission is always optically thin $\left(\tau_{850 \mu \mathrm{m}} \lesssim 0.0002\right)$. If we were to account for decreased dust heating in shielded regions, or the effect of growing ice mantles on the far-IR dust opacity, this might degrade the correlation between dust continuum intensity and surface density, but it seems unlikely that these effects would be large enough to alter significantly the filament widths inferred from far-IR dust emission, at least for typical molecular cloud environments.

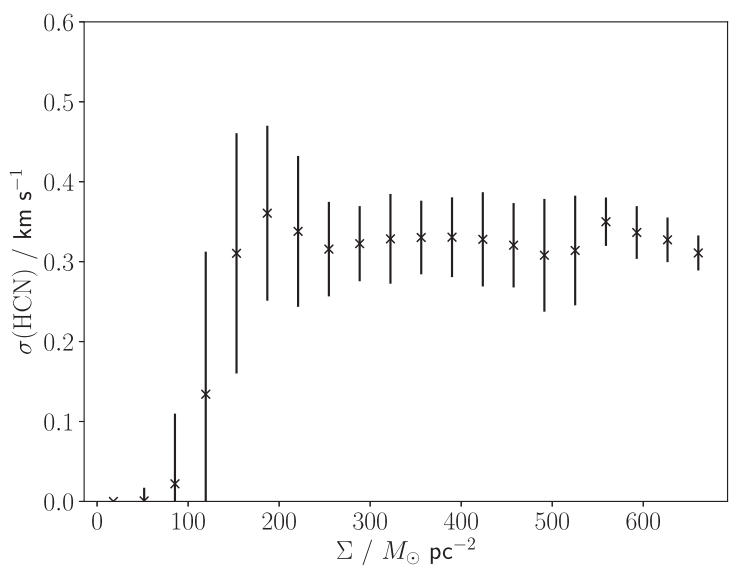

Figure 9. Radial velocity dispersion versus surface density for the $G=1$ case at $0.868 \mathrm{Myr}$, using ${ }^{13} \mathrm{CO}$ (left) and $\mathrm{HCN}$ (right). The points give mean values and the error bars give standard deviations. 

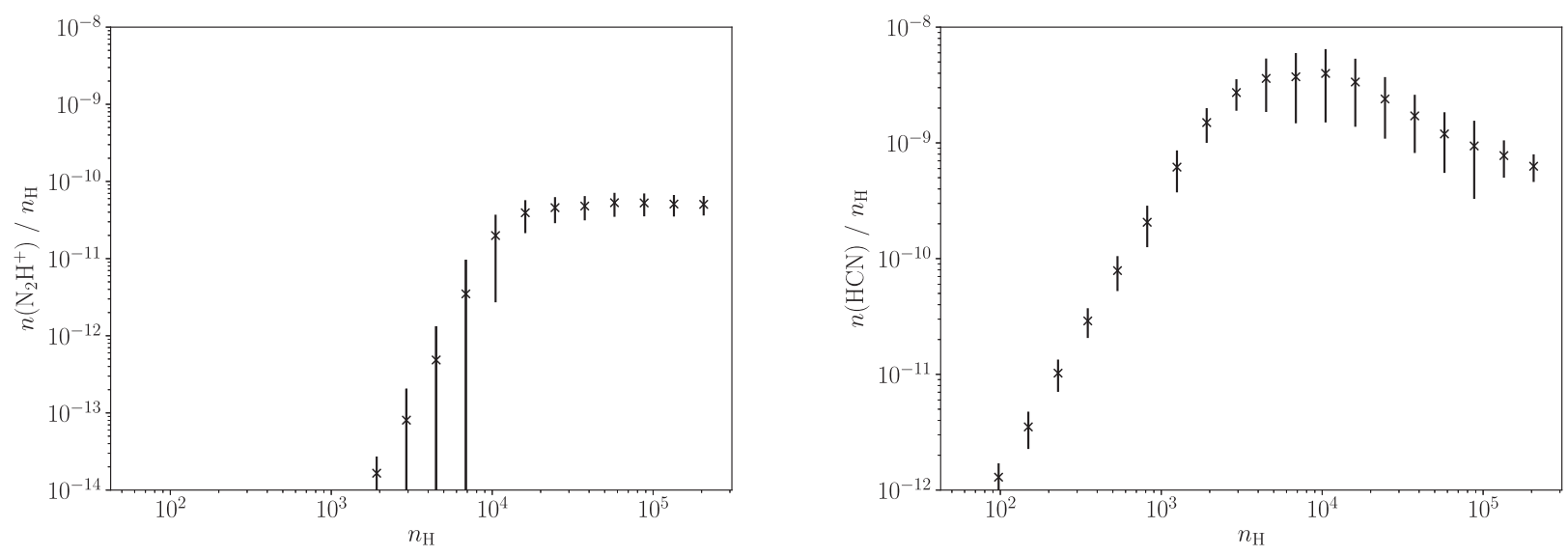

Figure 10. Gas-phase molecular abundances versus the volume density of hydrogen in all forms $\left(n_{\mathrm{H}}\right)$ for the $G=1$ case at $0.868 \mathrm{Myr}$ Left: $\mathrm{N}_{2} \mathrm{H}^{+}$. Right: $\mathrm{HCN}$. The points give mean values, and the error bars give standard deviations.
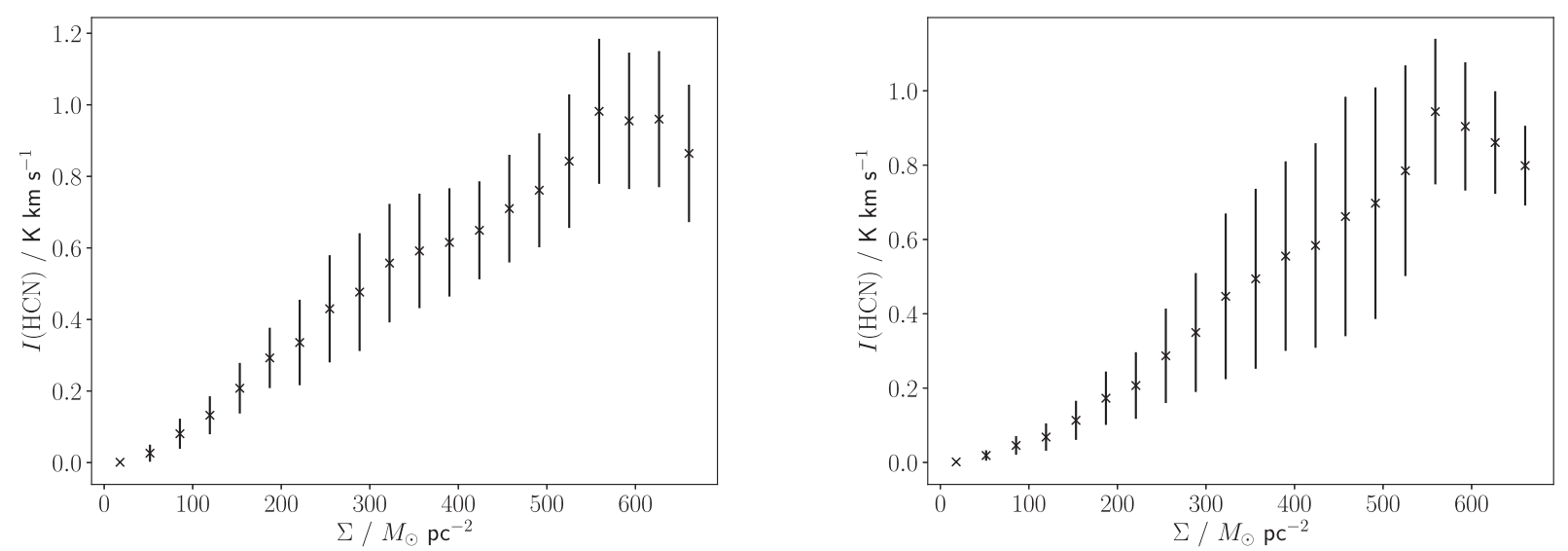

Figure 11. The ratio of intensity to surface density on the $y=0$ plane for the $G=1$ case at $0.868 \mathrm{Myr}$. Left: integrated line intensity of $\mathrm{HCN} J=1-0$ emission Right: integrated line intensity of HCN $J=1-0$ emission assuming a constant abundance of $3 \times 10^{-9}$. The points give mean values, and the error bars give standard deviations.

Moreover, the filament widths derived using molecular-line intensities, for which we do account for the effect of environment (gas-phase abundance, optical depth, etc.), are generally in good agreement with those seen observationally. CO isotopologues tend to return larger widths, unless the ambient FUV radiation field is strong (as, for example, in Orion). Molecules like $\mathrm{N}_{2} \mathrm{H}^{+}$, which have high critical excitation densities, typically return widths similar to, or even lower than, those inferred from far-IR dust emission. While our treatment of the FUV radiation field is crude, altering the strength from zero to five times the local value does not qualitatively change our conclusions. Thus, our results are broadly consistent with the universal characteristic filament width identified by Arzoumanian et al. (2011) and Arzoumanian et al. (2019).

Since we have calculated the molecular abundances selfconsistently with the hydrodynamics, we can evaluate the accuracy of the abundance prescriptions used by previous authors. Fig. 10 shows how the abundances of $\mathrm{N}_{2} \mathrm{H}^{+}$and $\mathrm{HCN}$ vary with the volume density of hydrogen in all forms,

$n_{\mathrm{H}}=2 n_{\mathrm{H}_{2}}+n_{\mathrm{H}^{\mathrm{o}}}+n_{\mathrm{H}^{+}}+\ldots$,

where the additional unspecified terms on the right-hand side of equation (4) represent molecules (e.g. $\mathrm{H}_{2} \mathrm{O}, \mathrm{NH}_{3}$, etc.), which are so rare that their contributions to the sum can safely be neglected. Smith et al. (2012) and Smith et al. (2013) assume constant abundances for both $\mathrm{N}_{2} \mathrm{H}^{+}$and $\mathrm{HCN}$, and $\mathrm{N}_{2} \mathrm{H}^{+}$does indeed have a roughly constant abundance at densities above $\sim 10^{4} \mathrm{~cm}^{-3}$, albeit somewhat smaller than the value adopted by Smith et al. (2012). However, the abundance of $\mathrm{HCN}$ is not even approximately constant over any significant density range; in particular, the abundance decreases by a factor of $\sim 5$ between $n_{\mathrm{H}} \sim 10^{4} \mathrm{~cm}^{-3}$ and $n_{\mathrm{H}} \sim 10^{5} \mathrm{~cm}^{-3}$ and probably continues to decrease at even higher densities. As the HCN (1-0) transition is typically optically thick at these densities, the decrease in abundance may not greatly affect the resulting line intensities, but the large change in abundance around the effective excitation density of $8 \times 10^{3} \mathrm{~cm}^{-3}$ (the density required to produce a line intensity of $1 \mathrm{~K} \mathrm{~km} \mathrm{~s}^{-1}$; Shirley 2015) may have greater effects. We find that assuming a constant HCN abundance of $3 \times 10^{-9}$ (Smith et al. 2012) reduces the absolute value of, and increases the scatter in, the line intensity as a function of column density, shown in Fig. 11, although as this is only by a modest factor $(\sim 1.5)$, it may not be of great importance. This, however, is not necessarily the case for all molecules, or for differing values of the radiation field and other input parameters, which can significantly affect the gas-phase abundances. In any case, $\mathrm{HCN}$ emission does not become optically thick until a surface density of $\sim 550 \mathrm{M}_{\odot} \mathrm{pc}^{-2}$, compared to around $200 \mathrm{M}_{\odot} \mathrm{pc}^{-2}$ for ${ }^{13} \mathrm{CO}$. For a cloud depth of $1 \mathrm{pc}$, this corresponds to 
an average volume density of $\left\langle n_{\mathrm{H}}\right\rangle=1.6 \times 10^{4} \mathrm{~cm}^{-3}$, two orders of magnitude below the critical density, and within a factor of a few of the effective excitation density from Shirley (2015). Recent work by Evans et al. (2020) has found that the majority of HCN emission does not actually appear to originate from dense gas, corroborating this conclusion.

While we have demonstrated that a simple physical model of an isolated turbulent cloud is consistent with the observed properties of molecular filaments, it remains to be seen whether more complex models of molecular clouds also have these properties. Federrath (2016) find that the inclusion of magnetic fields does not significantly affect the properties of filaments formed in their simulations. However, dynamically important magnetic fields can have a major effect on the abundances of key molecules, particularly in the densest regions (Tassis et al. 2012; Priestley et al. 2018, 2019). Other models of filament formation, such as cloud-cloud collisions (e.g. Balfour et al. 2015), will likely show similar chemical evolution, but the different kinematic structures involved could generate readily observable statistical differences in position-velocity maps based on molecular-line emission. We intend to investigate these possibilities in future work.

\section{CONCLUSIONS}

We have post-processed hydrodynamical simulations of an isolated turbulent isothermal molecular cloud, using a time-dependent chemical network and a radiative transfer model. Our main conclusions are as follows:

(i) The resulting filamentary structures have a characteristic width of $\sim 0.1 \mathrm{pc}$, which is clearly defined both on maps of the actual surface density and on maps of $850 \mu \mathrm{m}$ dust continuum emission.

(ii) Filament widths determined from $\mathrm{CO}$ isotopologues are several times larger than $0.1 \mathrm{pc}$, because, at surface densities $\Sigma \gtrsim$ $250 \mathrm{M}_{\odot} \mathrm{pc}^{-2}$, the line intensity is poorly correlated with the surface density.

(iii) Molecules used to trace dense gas, such as $\mathrm{N}_{2} \mathrm{H}^{+}$and $\mathrm{HCN}$, typically give filament widths similar to, or smaller than, those measured directly from the surface density.

(iv) This is broadly consistent with current observational data and suggests that the characteristic width identified by Arzoumanian et al. (2011) is a real physical phenomenon.

(v) Velocity dispersions within filaments are transsonic $\left(\sim 0.35( \pm 0.05) \mathrm{km} \mathrm{s}^{-1}\right)$ and roughly constant, up to the highest densities that our simulations reach.

(vi) We find evidence for significant radial velocity differences $\left(\sim 0.4 \mathrm{~km} \mathrm{~s}^{-1}\right)$ across filaments, and we suggest that this may be a useful discriminatory signature of turbulent filament formation.

(vii) We confirm that $\mathrm{HCN}$ may not be a reliable tracer of dense gas, because its (1-0) transition becomes optically thick at surface densities well below those expected based on its critical density of $\sim 10^{6} \mathrm{~cm}^{-3}$.

\section{ACKNOWLEDGEMENTS}

We are grateful to the referee for several constructive suggestions. We thank Paul Clark, Liz Watkins, Ollie Lomax, Niall Jeffrey, and James Wurster for their assistance with various aspects of this paper. FDP and APW acknowledge the support of a consolidated grant (ST/K00926/1) from the UK Science and Technology Facilities Council (STFC).

\section{DATA AVAILABILITY}

The data underlying this article will be shared on request. The codes used are all publicly available at: phantomsph.bitbucket.io (PHANTOM); uclchem.github.io (UCL_CHEM); github.com/lime-rt/lime (LIME).

\section{REFERENCES}

Aikawa Y., Herbst E., Roberts H., Caselli P., 2005, ApJ, 620, 330

André P. et al., 2010, A\&A, 518, L102

Arzoumanian D. et al., 2011, A\&A, 529, L6

Arzoumanian D., André P., Peretto N., Könyves V., 2013, A\&A, 553, A119

Arzoumanian D., Shimajiri Y., Inutsuka S.-i., Inoue T., Tachihara K., 2018, PASJ, 70, 96

Arzoumanian D. et al., 2019, A\&A, 621, A42

Balfour S. K., Whitworth A. P., Hubber D. A., Jaffa S. E., 2015, MNRAS, 453, 2471

Beuther H., Ragan S. E., Johnston K., Henning T., Hacar A., Kainulainen J. T., 2015, A\&A, 584, A67

Bohlin R. C., Savage B. D., Drake J. F., 1978, ApJ, 224, 132

Brinch C., Hogerheijde M. R., 2010, A\&A, 523, A25

Clark P. C., Glover S. C. O., Klessen R. S., 2012, MNRAS, 420, 745

Clarke S. D., Whitworth A. P., Spowage R. L., Duarte-Cabral A., Suri S. T., Jaffa S. E., Walch S., Clark P. C., 2018, MNRAS, 479, 1722

Dobbs C. L., Glover S. C. O., Clark P. C., Klessen R. S., 2008, MNRAS, 389 , 1097

Duarte-Cabral A., Dobbs C. L., 2016, MNRAS, 458, 3667

Duarte-Cabral A., Dobbs C. L., 2017, MNRAS, 470, 4261

Evans, Kim K.-T., Wu J., Chao Z., Heyer M., Liu T., Nguyen-Lu'o'ng Q., Kauffmann J., 2020, ApJ, 894, 103

Federrath C., 2016, MNRAS, 457, 375

Franeck A. et al., 2018, MNRAS, 481, 4277

Glover S. C. O., Clark P. C., 2012, MNRAS, 421, 116

Habing H. J., 1968, Bull. Astron. Inst. Netherlands, 19, 421

Hacar A., Tafalla M., Forbrich J., Alves J., Meingast S., Grossschedl J., Teixeira P. S., 2018, A\&A, 610, A77

Haworth T. J. et al., 2015, MNRAS, 450, 10

Holdship J., Viti S., Jiménez-Serra I., Makrymallis A., Priestley F., 2017, AJ, 154,38

Howard A. D. P., Whitworth A. P., Marsh K. A., Clarke S. D., Griffin M. J., Smith M. W. L., Lomax O. D., 2019, MNRAS, 489, 962

Kirk H., Myers P. C., Bourke T. L., Gutermuth R. A., Hedden A., Wilson G. W., 2013, ApJ, 766, 115

Kirk H., Klassen M., Pudritz R., Pillsworth S., 2015, ApJ, 802, 75

Larson R. B., 1981, MNRAS, 194, 809

Lee H.-H., Roueff E., Pineau des Forets G., Shalabiea O. M., Terzieva R., Herbst E., 1998, A\&A, 334, 1047

Matsumoto T., Dobashi K., Shimoikura T., 2015, ApJ, 801, 77

McElroy D., Walsh C., Markwick A. J., Cordiner M. A., Smith K., Millar T. J., 2013, A\&A, 550, A36

Orkisz J. H. et al., 2019, A\&A, 624, A113

Ossenkopf V., Henning T., 1994, A\&A, 291, 943

Panopoulou G. V., Tassis K., Goldsmith P. F., Heyer M. H., 2014, MNRAS, 444,2507

Panopoulou G. V., Psaradaki I., Skalidis R., Tassis K., Andrews J. J., 2017, MNRAS, 466, 2529

Peñaloza C. H., Clark P. C., Glover S. C. O., Klessen R. S., 2018, MNRAS, 475,1508

Peretto N. et al., 2013, A\&A, 555, A112

Price D. J., 2007, PASA, 24, 159

Price D. J. et al., 2018, PASA, 35, e031

Priestley F. D., Viti S., Williams D. A., 2018, AJ, 156, 51

Priestley F. D., Wurster J., Viti S., 2019, MNRAS, 488, 2357

Ragan S. E., Heitsch F., Bergin E. A., Wilner D., 2012, ApJ, 746, 174

Ragan S. E., Henning T., Tackenberg J., Beuther H., Johnston K. G., Kainulainen J., Linz H., 2014, A\&A, 568, A73 
Ragan S. E., Henning T., Beuther H., Linz H., Zahorecz S., 2015, A\&A, 573, A119

Sánchez-Monge Á. et al., 2014, A\&A, 569, A11

Schöier F. L., van der Tak F. F. S., van Dishoeck E. F., Black J. H., 2005, A\&A, 432, 369

Shirley Y. L., 2015, PASP, 127, 299

Smith R. J., Shetty R., Stutz A. M., Klessen R. S., 2012, ApJ, 750, 64

Smith R. J., Shetty R., Beuther H., Klessen R. S., Bonnell I. A., 2013, ApJ, 771,24

Smith R. J., Glover S. C. O., Klessen R. S., 2014, MNRAS, 445, 2900
Smith R. J., Glover S. C. O., Klessen R. S., Fuller G. A., 2016, MNRAS, 455, 3640

Smith R. J. et al., 2020, MNRAS, 492, 1594

Sousbie T., 2011, MNRAS, 414, 350

Suri S. et al., 2019, A\&A, 623, A142

Tassis K., Willacy K., Yorke H. W., Turner N. J., 2012, ApJ, 753, 29

Watkins E. J., Peretto N., Marsh K., Fuller G. A., 2019, A\&A, 628, A21

Whitworth A. P., Ward-Thompson D., 2001, ApJ, 547, 317

This paper has been typeset from a $\mathrm{T}_{\mathrm{E}} \mathrm{X} / \mathrm{LT} \mathrm{E} \mathrm{X}$ file prepared by the author. 